\title{
Notational Representation of Sign Language: A Structural Description of Hand Configuration
}

\author{
Jintae Lee \\ University of Aizu, Aizu-Wakamatsu City, Fukushima 965-80, Japan
}

\begin{abstract}
A universal description method of sign language is the most fundamental requirement in sign language research. Although a number of methods including notations have been invented, few are in substantial use for describing general sign languages. In this paper, we present structural description method of hand configuration based on human hand structure and geometric sign features, and explain with a series of arguments that this method has potential as a universal description method of sign language.
\end{abstract}

\section{Introduction}

Although, there are hundreds of sign languages in the world developed in various cultures, there is no agreed way of representing sign language. The question ' $\mathrm{Can}$ we develop a compact universal description method of sign language which provides conceptually tractable information of the signs?' is the most fundamental question in sign language research.

Three main methods can be identified in approaches to sign language representation. The first and the simplest is to use sign image itself. The image can then be edited or digitized to be available for applications. The difficulty is that this scenario involves an explosive amount of data, most of which would probably be useless in any given investigation. Techniques of pattern recognition and scene analysis may be used extract necessary information from the image; however, they have not been developed to this level of practical use.

The second is to describe signs in natural language. The problem is now the reverse of that for imagery: the description may be compact; but actual regeneration of the signs requires a certain amount of knowledge of the natural language. Moreover, natural language descriptions are subject to ambiguity and imprecision in specifying positions, traces, and styles of hand motion.

The third is to describe signs in a notation or symbolic form. This method is the most feasible because it abstracts geometric information of the sign in a more compact and clearer way than the other two.

The expressive power of notation can be exemplified by musical score. $\mathrm{Mu}-$ sical score is so well-designed as a description of music that it is used in every process of musical composition: in recording and refining musical ideas, and in performing music.

Argument 1 As a form of sign language representation, notation is superior to natural language or image in compactness and clearness. 
Our further discussion in this paper is organized as follows: Section 2 surveys conventional sign language notations. Section 3 introduces structural description method of hand configuration. Section 4 gives comparison of the method with other notations. Section 5 concludes this paper.

\section{Conventional Sign Language Notations}

Two notation systems, Stokoe notation [1] and Honna notation [2], which are known to the general public through books and thus provide sufficient information are chosen to be examined in this section.

\subsection{Stokoe Notation}

Stokoe notation, the world's first practical notation system of sign language, has been developed in 1964 by William C. Stokoe and his colleagues to describe separate signs of American Sign Language (ASL) by symbols. Analogous with the phoneme of natural language, Stokoe named chereme (CARE-eem, the first syllable from a Homeric Greek word meaning 'handy') the unit of sign language [3]. Cheremes are instances of three sign aspects which distinguish a sign from all other signs in the language: (1) the place where it is made, (2) the distinctive configuration of the hand or hands making it, and (3) the action of the hand or hands.

Stokoe used fifty-five symbols to describe about 3,000 signs of ASL [1]. A sign is written as $T D^{s}$, where ' $T$ ', ' $D$ ', and ' $s$ ' represent tab (tabular, the place), dez (designator, the active hand), and sig ( signation, the action) symbol, respectively. Signs are written like $T D_{s}^{s}$ if the sig actions are combined; that is they are done at the same time. The sig symbols are written side by side $\left(T D^{s s}\right)$ if one sig action is done first and a second follows. Two dez symbols $\left(T D D^{s}\right)$ are written if both the signer's hands serve as a double dez.

\subsection{Honna Notation}

Stokoe notation stimulated researchers of sign language to extend the idea to their own sign languages. In 1984, Honna et al. developed a notation system suited to Japanese Sign Language (JSL) [2].

Honna notation also regards sign language as being composed of three aspects, i.e. hand shape, movement, and location. In Honna notation, however, hand shape is again decomposed into two independent elements, i.e. hand configuration and orientation. Hand configuration is further classified into basic form $(H)$ and transconfigurations $\left(C, B, B^{\prime}, P, A, T, V\right)$. Orientation is composed of two directions, i.e. the direction of fingertips (to be exact, it must be the direction of the middle finger when the hand is open) and the direction of the

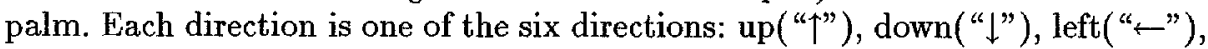
$\operatorname{right}($ " $\rightarrow$ "), forward $(+)$, and backward(-). Movement and location are classified into subgroups. 


\subsection{Review of the Notations}

Honna notation is more general than Stokoe notation and can describe hand shapes in JSL and ASL. Unfortunately, however, even Honna notation is probably insufficient as a universal sign language notation because it does not reflect real hand configuration correctly. The finger configurations are defined ambiguously, and constraints of the human hand that exert delicate influence upon hand configuration are not taken into account. Consequently, correct hand shape cannot be deduced from the notation.

Argument 2 The hand configuration should be described in terms of global features so that the description is not sensitive to local deformation. At the same time, it should be described with reasonable accuracy so that unrealistic hand configurations are not generated.

"Structural" notation based on anatomical structure of the human hand and structural analysis of signs can overcome the drawback of the conventional notations.

\section{Structural Description of Hand Configuration}

In this section, we describe a structural description method of hand configuration (hand shape) based on mechanical peculiarities and internal constraints of the human hand. It has been incorporated into the design of "Handnotation" of which we have been working on the development as a general sign language notation.

\subsection{Cheremes Concept}

We accept Stokoe's concept that cheremes are derived from three aspects of signs, i.e. hand configuration, location, and movement. However, we note the qualitative difference of the three aspects of sign (Fig. 1): Hand configuration and location are static in nature, but movement is dynamic. The number of distinguishable hand shapes is finite, while that of location or movement is finite or infinite. We also believe that movement is nothing but change of static aspects. Therefore, not only change of location but also change of hand configuration is a movement for us.

Argument 3 Chremes are derived from three aspects of sign: two static aspects - hand configuration and location, and one dynamic aspect-movement. Movement is change of static aspects in a time interval.

\subsection{Hand Configuration Analysis}

We take a hand configuration to be a set of feature specifications. 
Definition 1. A configuration is a set of feature specifications. A feature specification is an ordered pair of the form <feature-name, feature-value $>$, where a feature name is an atomic symbol begins with capital letter and a feature value is an atomic symbol in small letter or a symbol indicating a part of the hand or a configuration. When $\langle f, v\rangle$ is a member of configuration $C$, we write $C(f)=v$, i.e. $C$ yields the value $v$ for the argument $f$.

Fig. 2 illustrates the skeleton of the right hand seen from the palmar side. Some terminology and abbreviations in this figure will be used to facilitate our discussion. For example, M(II) represents the metacarpal bone of the middle finger. Joint angle will be represented by the symbol $\theta_{\beta}^{\alpha}(\gamma)$, where $\alpha$ is the rotation axis, $\beta$ the joint, and $\gamma$ the finger.

Hand configuration (HC) is represented by five finger configurations (FIN(f), $\mathrm{f}=\mathrm{I}, \mathrm{II}, \mathrm{III}, \mathrm{IV}, \mathrm{V}$ ) and orientation configuration (ORI):

$$
\text { HC }=\{\text { FIN(I), FIN(II), FIN(III), FIN(IV), FIN(V), ORI }\}
$$

Considering that fingers I, II, III, and IV are planar manipulators [5], FIN(i) (i $=\mathrm{I}, \mathrm{II}, \mathrm{III}, \mathrm{IV}, \mathrm{V})$ can be specified by $\theta_{D I P}, \theta_{P I P}$, and $\theta_{M P}$ of finger $\mathrm{i}$. If we use the constraint between the joint angles of DIP and PIP $\left(\theta_{D I P}=\frac{2}{3} \theta_{P I P}\right)[5]$, bending state of a finger can be specified by $\theta_{M P}$ and $\theta_{P I P}$.

Although $\theta_{M P}$ and $\theta_{P I P}$ can have continuous values within the joint angle limits, the bending degree (feature FLEX) can be classified according to singularity:

1. $\theta$ has zero value.

2. $\theta$ has the maximum value within its joint angle limit.

3. $\theta$ has a value between zero and the maximum value.

4. $\theta$ is determined by other joint or finger(s).

These four classes are represented as feature values straight, bent, curved and passive, respectively. The MP joint can move left or right (feature ASIDE) and make the finger contact or cover the next finger. Thus, configuration of finger $i$ is the set of features:

$$
\begin{aligned}
\operatorname{FIN}(i) & =\{\operatorname{MPC}(i), \operatorname{PIPC}(i)\}, i=\mathrm{I}, \mathrm{II}, \mathrm{III}, \mathrm{IV} \\
\operatorname{MPC}(i) & =\{\mathrm{FLEX}, \operatorname{ASIDE}\}
\end{aligned}
$$

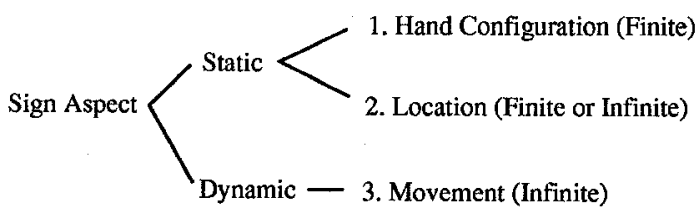

Fig. 1. Qualitative differences of three sign aspects. 


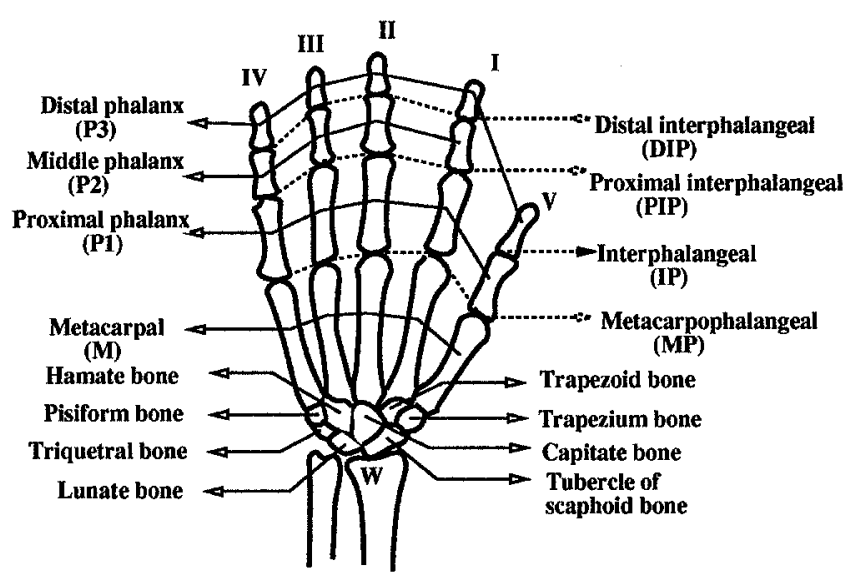

Fig. 2. The hand skeleton seen from the palmar side [4].

$$
\begin{aligned}
\operatorname{PIPC}(i) & =\{\text { FLEX }\} \\
\text { ASIDE } & =\{\text { no, CONTACT, COVER }\}, \text { where }
\end{aligned}
$$

(MPC(i) or PIPC(i))(FLEX) $\in$ \{straight, curved, bent, passive)(6) ASIDE(CONTACT or COVER) $\in\{$ I, II, III, IV, V $\}$

The feature CONTACT need not be specified when MPC(FLEX)= bent because the finger inevitably is in contact with its neighboring finger(s) when their MP joints are fully bent (See [5]).

Thumb configuration is more complex to describe since the thumb moves with larger degrees of freedom than other fingers, frequently touching other fingers or the palm. The thumb operates on hand configuration in two ways - by its own configuration or by its relationship with other fingers. When the thumb operates on hand configuration by its own configuration, its configuration is usually at a singular point within the whole configuration space of the thumb. When the thumb operates on hand configuration by its relationship with other fingers, it touches, covers or lies between finger(s).

$$
\mathrm{HC}(\mathrm{FIN}(\mathrm{V})) \in \mathrm{T} \text {, where }
$$

$\mathrm{T}=\{$ open, closed, stand, fold-in, fold-out, TOUCH, INBETWEEN $\}$

$$
\begin{aligned}
\operatorname{FIN}(\mathrm{V})(\text { TOUCH }) & =p \\
\operatorname{FIN}(\mathrm{V})(\mathrm{INBETWEEN}) & =\{\mathrm{F} 1, \mathrm{~F} 2\}
\end{aligned}
$$

where $p$ is the place touched and the values of $\mathrm{F} 1, \mathrm{~F} 2$ are finger numbers. 
Orientation configuration consists of two directions, i.e. the direction of $\mathrm{M}$ (II) (DIRM) and the direction normal to the palm (DIRP). It is known that eight directions are adequate to classify directions of shapes on a plane [6]. If we expand the plane into space, all the possible directions become 26 . Thus, the value of DIRM is specified as one of 26 unit vectors in our notation. When DIRM is decided, DIRP can have one of eight directions on the plane normal to DIRM.

$$
\begin{aligned}
\text { ORI } & =\{\text { DIRM, DIRP }\} \\
\text { ORI(DIRM) } & =\boldsymbol{e}_{i}, \text { where } i=1,2, \cdots, 26 \\
\text { ORI(DIRP) } & =\boldsymbol{f}_{j}, \text { where } j=1,2, \cdots, 8 .
\end{aligned}
$$

Example 1. The hand configuration of the hand in Fig. 4(a) would be the set of feature specifications as follows:

$$
\begin{aligned}
& \text { three }= \\
& \{<\mathrm{FIN}(\mathrm{I}),\{<\mathrm{MPC},\{<\mathrm{FLEX}, \text { straight }>,<\text { ASIDE, no }>\}>\text {, } \\
& <\text { PIPC, }\{<\text { FLEX, straight }>\}>\}>\text {, } \\
& <\text { FIN(II), }\{<\text { MPC, }\{<\text { FLEX, straight }>,<\text { ASIDE, no }>\}>\text {, } \\
& <\text { PIPC, }\{<\text { FLEX, straight }>\}>\}>\text {, } \\
& <\text { FIN(III), }\{<\text { MPC, }\{<\text { FLEX, curved }>,<\text { ASIDE, no }>\}>\text {, } \\
& <\text { PIPC, }\{<\text { FLEX, straight }>\}>\}>\text {, } \\
& <\text { FIN(IV), }\{<\text { MPC, }\{<\text { FLEX, passive }>\}>\text {, } \\
& <\text { PIPC, }\{<\text { FLEX, passive }>\}>\}>\text {, } \\
& <\text { FIN }(\mathrm{V}),\{<\text { TOUCH, IV }>\}>\text {, } \\
& <\text { ORI, }\{<\text { DIRM, } \mathrm{u}>,<\text { DIRP, } 0>\}>\}
\end{aligned}
$$

\subsection{Visualization}

As it has often been said that a picture is worth a thousand words, pictures can convey meaning in a more intuitive and understandable way than onedimensional, textual words. Taking this point into account, we created a visual notation in which the information of hand configuration expressed in feature notations can be visualized. The symbols (not all) are illustrated in Fig. 3. They are designed so that the meaning can be understood by their visual form. For instance, the orientation a DIRM symbol means can quickly be understood simply by imagining an arrow located in space. The symbols are written in corresponding places of "sign score" (Fig. 4(b)).

Argument 4 Symbols in sign language notation must be designed to reveal their meaning in their visual forms.

\section{Experiment}

Our method can describe all the manual letters of ASL, JSL, and KSL (Korean Sign Language). Compared with Stokoe and Honna notations, our notation describes more general hand configurations with fewer symbols and more readable 
A. Symbols for finger joints (except the thumb joints) configuration

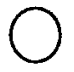

straight

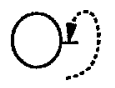

contact

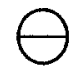

curved

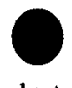

bent

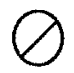

covering

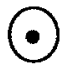

passive

B. Symbols for the thumb configuration

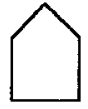

closed

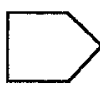

open

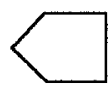

fold

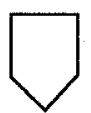

stand

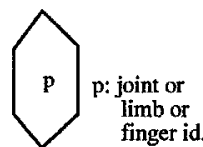

touch

\section{Symbols for ORIM}

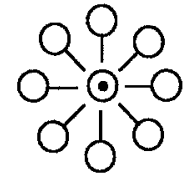

forward directions

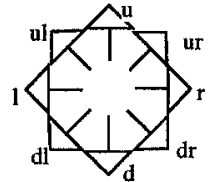

backward directions

D. Symbols for ORIP

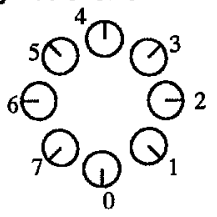

Fig. 3. Visual symbols for specifying hand configuration.

forms (Table 1). Furthermore, its description is more faithful to real hand shapes. For instance, while all the III configurations in their "basic forms" $H_{123}$ (manual letter for " 3 "),$H_{1234}$, and $H_{3}$ are described identically in Honna notation, we describe the III configuration in the manual letter for " 3 " as "curved" on MP(III) rather than "straight" considering constraints caused by finger IV (Fig. 4).

\section{Conclusion}

We presented a structural description method of hand configuration based on anatomical structure and constraints of the human hand. We have shown that this description is compact and can describe more general and precise hand configurations than conventional empirical notations.

There are more works to be done on describing sign movement aspect. As our structural approach to hand configuration description has been very encouraging, we are working to extend it to general sign motion description.

\section{References}

1. Stokoe, W.: A Dictionary of American Sign Language on Linguistic Principles. Linstok Press (1976)

2. Kanda, W.: Yubimoji no Kenkyu. Koseikan (1986) (in Japanese)

3. Stokoe, W: Semiotics and Human Sign Languages. Mouton Publishers (1972)

4. Pernkopf, E.: Pernkopf Anatomy, vol. 2., 3 ed. Uran \& Schwarzenberg (1989) 
Table 1. Comparison of notations.

\begin{tabular}{|l||c|c|c|}
\hline Notation & Stokoe Notation & Honna Notation & Handnotation \\
\hline Features & conceptual & conceptual & geometric \\
\hline $\begin{array}{l}\text { Low level unit } \\
\text { of description }\end{array}$ & whole hand & finger & joint \\
\hline $\begin{array}{l}\text { Number of } \\
\text { basic symbols }\end{array}$ & $\begin{array}{c}\text { Dez: } 19 \\
\text { (Tab: } 12) \\
\text { (Sig: } 24)\end{array}$ & $\begin{array}{c}\text { hand config.: } 9 \\
\text { hand orient.: } 6\end{array}$ & $\begin{array}{l}4 \text { fingers config.: } 6 \\
\text { thumb config.: } \\
\text { hand orient.: } 26\end{array}$ \\
\hline $\begin{array}{l}\text { Describable } \\
\text { config. }\end{array}$ & $\begin{array}{c}\text { limited } \\
(19)\end{array}$ & $\begin{array}{c}\text { general but limited } \\
\text { in orientation }\end{array}$ & general \\
\hline $\begin{array}{l}\text { Form } \\
\text { Faithfulness to } \\
\text { real hand shapes }\end{array}$ & weak & weak & visual \\
\hline Constraints & not considered & not considered & considered \\
\hline
\end{tabular}

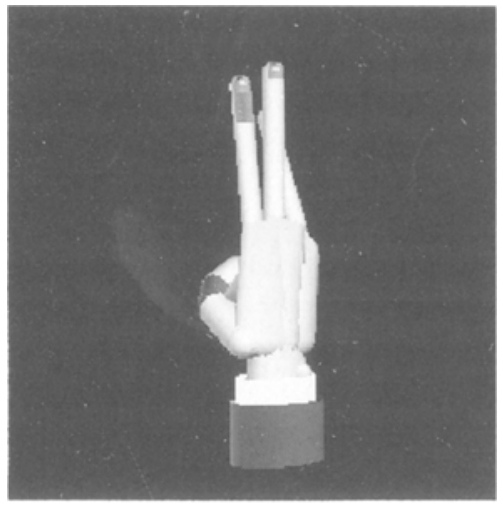

a.

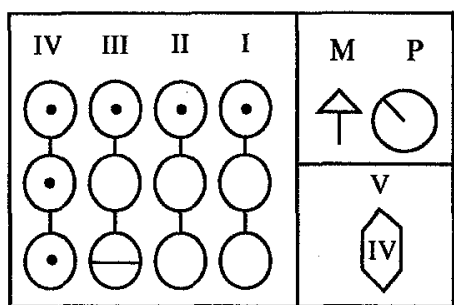

b.

Fig. 4. Manual letter for " 3 " drawn by the Handnotation interpreter (a), and its notational description (b). III is "curved" on MP by constraints caused by IV.

5. Lee, J., Kunii, T.: Constraint-based hand animation. Models and Techniques in Computer Animation, Springer-Verlag (1993) 110-127

6. Nishida, H., Mori, S.: Algebraic description of curve structure: IEEE PAMI, 14:5 (1992) 516-533

7. Lee, J., Kunii, T.: Generation and recognition of sign language using graphic models: Proc. ACMJ/IISF Int. Symposium on computers as our better partners, World Scientific (1994) 96-103 